\title{
The future of suspension rheophysics: comments on the 2008 workshop
}

\author{
Philippe Coussot • Norman J. Wagner
}

Received: 24 July 2009 / Accepted: 24 July 2009 / Published online: 5 August 2009

(C) Springer-Verlag 2009

This volume of Rheologica Acta contains manuscripts from contributors to the Workshop on Rheophysics held in January 7-9, 2008, at the ESPCI, Paris. It was organized by P. Coussot, P.H. Jézéquel (Lafarge), and F. Lequeux (ESPCI) within the frame, and with the financial support, of the Lafarge-Ecole des Ponts ParisTech-Ecole Polytechnique Chair "Material Science for Sustainable Development." The aim of this Chair (http://chaire-lafarge.enpc.fr/) is to foster exchanges between the academics potential in emerging fields and the growing needs for interdisciplinary scientific approaches for applications in civil and environmental engineering. The workshop was attended by over 85 participants from six countries, including academic faculty and students as well as industrial scientists. Herein, we briefly summarize some of the key contributions so as to provide the reader with both a guide to the work as well as a brief summary of some challenges identified for future research.

Suspensions are ubiquitous in the chemical and materials processing industry, and as scientists from Lafarge note, controlling suspension rheology is the key to the largest by weight industrial production, namely concrete. There are a few common issues that confront industrial scientists working with suspensions, namely that they are often very concentrated, they are in

P. Coussot $(\varangle)$

University Paris-Est, Insitut Navier,

Champs-sur-Marne, France

e-mail: Philippe.Coussot@lcpc.fr

N. J. Wagner

Department of Chemical Engineering,

University of Delaware, Newark, DE 19716, USA complex, multi-component formulations often evolving around rheology control, and industrial suspensions often have both a wide particle size distribution and many complex interactions that arise from a variety of different chemical surfaces, solvent mixtures, and added surfactants, polymers, and salts. In addition, these complex mixtures often contain broad distributions of timescales, where both chemical and physical aging are possible. As noted, formulation always, to some level, addresses rheology, where once an acceptable level of viscosity control is achieved, other nonlinear effects such as normal forces, shear migration, shear banding, sedimentation, thixotropy, aging and shear thickening, and jamming may complicate or frustrate the application. The contributions herein provide insight into understanding all of these complications through model system studies and modeling as well as identify key areas needing further research.

For example, Toussaint reviews the impact of the formulation changes on the rheological properties of well-controlled cement pastes. In particular, it is striking to see how subtle changes in the particle or additive characteristics may have an impact on these properties and in particular the shear-thickening effect. Also, Toussaint shows that it is possible in a practical industrial problem to use the existing theories developed for model materials to both understand and improve the rheology of industrial products.

The main origin of the complex rheology arising in these concentrated systems is the close proximity of the particles immersed in a suspending medium that gives rise to a very complex hydrodynamic field, which leads to the possibility of collective effects and couplings between flow and microstructure. Morris reviews the fundamental aspects of flow-microstructure coupling 
in model suspensions and provides new keys for understanding complex suspension rheology phenomena such as shear-thinning, shear-thickening, normal stress differences, as well as phenomenon such as shearinduced migration.

Shear thickening is indeed one of the more spectacular rheological effects associated with concentrated suspensions and remains a current topic of investigation. The work of Kalman describes the novel method of flow-ultra small angle neutron scattering and its application to measuring the statistical properties of the concentration fluctuations underlying shear thickening, i.e., the structure of "hydroclusters." Understanding how the microstructural transition of hydrocluster formation drives the macroscopic shear-thickening response provides a method for controlling the phenomenon. Hébraud discusses the specific case of "catastrophic" shear thickening associated with a jamming of the structure at a critical shear rate. It is demonstrated that very large stress fluctuations can be observed, along with a change of the sign of normal stress and dilatancy. Clearly, such behavior is associated with microstructural transitions, but what constitutes transition from continuous shear thickening to dilatancy remains a challenging problem.

The above works focused on model systems in which colloidal interactions were initially negligible or moderated by appropriate physicochemical treatment. Thus, the theoretical analysis of the observed effects could rely on a description of the material as made of individual particles evolving more or less collectively. The work of Osuji shows an even more complex shearthickening effect in colloidal suspensions with attractive interactions and floc structures. Shear thickening is suggested to be due to a breakage of flocs, which increases with shear rate and which finally gives rise to larger hydrodynamic dissipation as the smaller particles are now dispersed in the liquid. Direct visual observations of vorticity-aligned structures on longer length scales vividly illustrate the complex microstructures forming in such model suspensions in a relatively simple flow. Understanding these hierarchical structural rearrangements, their coupling to the flow, and their effect on the rheology finally relates to more ancient and still vivid significant challenges in the field of colloidal suspensions, which have impact for understanding of the physical origin of their shear thinning, yielding, or thixotropic behavior.

Another important complementary work is that of Lemaitre, who provides an original review of the problem of the rheophysics of concentrated suspensions from the point of view of dry granular flow properties. This field has indeed emerged rather recently (about
25 years ago), and there is now a significant understanding. In fact, there are obviously strong similarities in concentrated suspensions and dry granular materials since they both contain a high concentrated of particles in a limited volume. On the basis of a rheophysical approach, focused on dimensional or scaling analysis (in particular comparing the amount of main energy dissipations), Lemaitre et al. nevertheless clarify the physical origin of the analogies and the transition of regimes in the different situations. In particular, they show how a regime in which inertial effects are predominant may occur even in the presence of significant contact forces up to the jamming transition.

Another critical example of consequence of the flowmicrostructure coupling is provided by Snabre, who shows that such effects can occur even within a concentrated suspension at rest during sedimentation. Snabre presents a very detailed, original ParticleImage-Velocimetry study of the temporal microstructure evolution of a granular suspension at rest, which he can correlate to size segregation and sedimentation effects such as jamming. This study provides a unique and thorough approach to study the link between local and collective effects in concentrated suspensions under gravitational settling, which suggests several implications for the properties of shear flow of similar systems.

Finally, another dramatic effect observed in flowing suspensions is discussed by Ovarlez, namely shear banding. This effect has been widely described for micellar solution, but in that case, it is likely associated with a phase transition. Here, Ovarlez et al. focuses on this effect in the case of colloidal or non-colloidal suspensions. In particular, they show that this effect is a consequence of temporal structure evolution in flowing pastes and suspensions and emphasize that it is critical to distinguish true shear banding from the simpler effect of coexisting static solid and flowing liquid regions in yield stress fluids. They suggest, from a global point of view, that shear banding in these systems has a common physical origin: two physical phenomena are in competition, and depending on the flow conditions, one of them becomes dominant and makes the system evolve in a specific direction. Further, by reviewing observations across a broad spectrum of complex fluids, they conclude that shear banding may be more the rule than the exception.

These reports of current research advances in suspension rheophysics all focus to varying extents on the underlying connection between microstructure and macroscopic behavior. This is facilitated by advances in experimental methods, such as rheo-SANS and light microscopy. In addition, a number of investigations 
highlight the need to also directly measure the local kinematics, which can be very complex even for seemingly simple geometries, by such methods as rheoNMR and PIV. This highlights the need for evermore precise and robust measurement methods where microstructure, kinematics, and bulk properties can be determined simultaneously or at least in a complementary manner to enable advances in our understanding of model systems. Robust theory and simulations are shown to be essential tools to provide a quantitative link between these observations and place specific model systems (hopefully) into the context of a broader, more universal behavior. Note also that all of these investigations to some extent treat systems that exhibit solid-liquid and/or flowing-jamming transitions, illustrating the tremendous interest (and hence, our lack of a complete understanding), both academic and practical, in such behavior and its consequences. It is reasonable to expect that significant advances in our ability to formulate and control the rheological properties of products based on concentrated suspensions and pastes will come through experimental investigations on model systems that determine microstructure and kinematics simultaneously with bulk properties. Ideally, these experimental will be analyzed with particlelevel modeling connecting particle properties and interactions to microstructure and stresses, which becomes a microscopic constitutive equation for macroscopic modeling of the suspension kinematics. This remains a challenge and perhaps a speculation of a roadmap for future work.
We invite the interested reader to draw their own conclusions after reading these insightful contributions, as well as identify fruitful areas for future research. Note that our summary is necessarily brief and, therefore, incomplete as well as biased by our own research interests-for this, we take full responsibility. Nevertheless, we hope you will benefit from these collected contributions as well from this introduction. In closing, we mention three very recent topical reviews of possible interest to the reader that provide overviews of the current literature on the microstructure of suspensions (Vermant and Solomon 2005), the modeling of pastes (Coussot 2007), and thixotropy (Mewis and Wagner 2009a), as well as one additional attempt to summarize recent advances in suspension rheology (Mewis and Wagner 2009b). We thank the sponsors and organizers of this workshop as well as the editors of Rheologica Acta for their dedicated effort to create this compendium of current research manuscripts in suspension rheophysics.

\section{References}

Vermant J, Solomon MJ (2005) Flow-induced structure in colloidal suspensions. J Phys, Condens Matter 17(4):R187R216

Coussot P (2007) Rheophysics of pastes: a review of microscopic modelling approaches. Soft Matter 3(5):528-540

Mewis J, Wagner NJ (2009a) Thixotropy. Adv Colloid Interface Sci 147-148:214-227

Mewis J, Wagner NJ (2009b) Current trends in suspension Rheology. J Non-Newton Fluid Mech 157:147-150 